\title{
Etiology, Antibiogram and Quantitative Endotracheal Aspirate Cultures in Ventilator Associated Pneumonia Patients in a Tertiary Care Hospital
}

\author{
Mir Salman Ali, Sreekanth Basireddy ${ }^{*}$, Manisha Singh and Vasanti Kabra
}

Department of Microbiology, Kurnool Medical College, Kurnool, Andhrapradesh, India

*Corresponding author

\begin{tabular}{|l|}
\hline Ke y w o r d s \\
Ventilator associated \\
$\begin{array}{l}\text { Pneumonia (VAP) } \\
\text { Nosocomial infection }\end{array}$ \\
\hline Article Info \\
\hline $\begin{array}{l}\text { Accepted: } \\
\text { 04 June } 2019 \\
\text { Available Online: } \\
\text { 10 July } 2019\end{array}$ \\
\hline
\end{tabular}

\section{Keywords}

Ventilator associated Pneumonia (VAP) Nosocomial infection

$$
\text { predo }
$$
organism isolated was Acinetobacter baumanii $(31.3 \%)$ followed by Klebsiella pneumoniae $(24.5 \%)$. In early onset VAP Klebsiella pneumonia was the most common while in late onset Acinetobacter baumanii was the most common. Multidrug resistance was common in all the isolates with Acinetobacter baumanii topping the list. Carbapenem resistance was observed in $40.6 \%$ of Acinetobacter baumanii whereas in K. pneumoniae it is only $12 \%$. Polymyxin B and colistin are the most susceptible drugs for Acinetobacter and Pseudomonas whereas in enterobacteriace (E. coli and $K$. pneumoniae) tigecycline was the best drug ( $0 \%$ resistance) followed by Imipenem $(12 \%$ and $13.3 \%$ resistance respectively). Among S. aureus, Methicillin resistance was observed in $66.6 \%$ of cases all the S. aureus were sensitive to vancomycin, linezolid and tigecycline. Conclusion, Ventilator associated pneumonia continuous to be an important challenge to the critical care physician as well as microbiologist both for treatment and diagnosis. A high index of clinical suspicion along with early and prompt diagnosis by using various parameters that include quantitative endotracheal cultures is of utmost importance in appropriate management of the patients, which further decrease the mortality and morbidity along with decrease in the cost associated with the treatment of these infections. 


\section{Introduction}

Hospital acquired infections are the biggest burden in terms of mortality, morbidity as well as economically in any healthcare setup. Ventilator associated Pneumonia is the second most common nosocomial infection in critically ill patients, affecting one fourth of all hospital acquired infections ${ }^{1}$. The mortality attributable to VAP has been reported to range between 0 and $50 \%{ }^{1,2}$.

The etiology of VAP primarily include organisms like Pseudomonas, Klebsiella Acinetobacter, Serratia, Enterobacter and Stenotrophomonas and MRSA etc, which are all multidrug resistant pathogens and augments the toll of mortality if not diagnosed and treated promptly.

Ventilator-associated pneumonia is defined as pneumonia occurring more than $48 \mathrm{hrs}$ after patients have been intubated and kept on mechanical ventilation. Diagnosing VAP requires a high clinical suspicion combined with bedside examination, radiographic examination, and microbiologic analysis of respiratory secretions, which involves a diverse group of specialized medical personnel including the microbiologist who plays a vital role in diagnosis of ventilator associated pneumonia, but it is always difficult to differentiate simple colonization of the endotracheal tube from significant infection. Though many invasive procedures like BAL (bronchoalveolar lavage), PSB (protected specimen brush samples) are having high sensitivity and specificity in the diagnosis, non-invasive and nonbronchoscopy techniques like quantitative endotracheal aspirate cultures, having advantages like less compromise of oxygenation, ventilation and respiratory mechanics during the procedure. Many studies conducted by different researchers have shown that quantitation of endotracheal aspirate is a promising tool in the diagnosis of ventilator associated pneumonia when the count $>10^{5} \mathrm{cfu} / \mathrm{ml}^{3,4}$.

Due to poor specificity and poor positive predictive value, non quantitative analysis will result in over diagnosis and therefore unnecessary treatment of VAP. Such an approach will result in excess antibiotic use with its attendant cost, potential toxicity, and selection of drug-resistant organisms.

To potentially improve the specificity of the diagnosis of VAP and the consequent unnecessary antibiotic use and its associated problems, the role of quantitative cultures of respiratory secretions is pivotal.

To diagnose ventilator associated pneumonia by using quantitative endotracheal aspiration cultures and to identify the bacterial pathogens and determine the antimicrobial susceptibility pattern of those pathogens.

\section{Materials and Methods}

A prospective study was done enrolling patients from the multidisciplinary ICUs of tertiary care hospital (SVS hospital, mahbubnagar) from October 2013 - July 2015

\section{Inclusion criteria}

Intubated patients (irrespective of age) who are mechanically ventilated for more than 48 hrs, with a clinical suspicion of pneumonia.

\section{Exclusion criteria}

\section{Patients with pre-existing pneumonia}

A total of 183 clinically diagnosed Ventilator Associated Pneumonia cases were selected and data such as name, age, gender, date of admission into Intensive care unit, chief complaints, risk factors involved and duration 
of mechanical ventilation were obtained. Data related to general physical examination, radiological and hematological investigations were collected.

\section{Specimen collection and processing}

Endotracheal aspirate (ETA) was collected under all aseptic precautions. A sterile 22 inch suction catheter was introduced into respiratory tract for a distance of $20-25 \mathrm{cms}$ and the specimen was aspirated into a sterile container. The sample was transported to the laboratory immediately for processing.

The ETA so collected was subjected to Gram's stain. The specimen was further diluted using sterile saline (1 in 100) and the dilution factor was noted (Fig. 1).

The specimen was then inoculated for quantitative culture using standard techniques onto MacConkey agar, Blood agar and Chocolate agar plates using a $4 \mathrm{~mm}$ sterile Nichrome loop (HiMedia), and then incubated at $37^{\circ} \mathrm{C}$. The plates were examined for growth at $24 \mathrm{hrs}$, if there was no growth, it was further incubated for another $24 \mathrm{hrs}$. The CFU/ml was then calculated, considering the dilution factor.

Bacterial growth with $>10^{5} \mathrm{CFU} / \mathrm{ml}$ was given significance as a pathogen, and was further identified using appropriate biochemical tests. The antibiotic susceptibility testing was performed according to the recommended CLSI procedures.

\section{Interpretation of results}

The diagnostic thresholds for endotracheal aspirate (ETA) were taken as $10^{5} \mathrm{CFU} / \mathrm{ml}$. Growth below the threshold was assumed to be due to colonization or contamination.,

Descriptive statistical analysis has been carried out in the present study Data was analyzed statistically by computing percentage, mean and standard deviation. Wherever necessary, the results are depicted in the form of graphs and charts.

\section{Results and Discussion}

A total of 183 suspected cases were included in the study. Majority of the patients were males $(60.1 \%)$ followed by females $(39.8 \%)$. The male to female ratio was 1.5:1 indicating the male predominance

Majority of the patients were in the age group of 21 to $40 \mathrm{yrs}(37.1 \%)$ followed by 41 to 60 yrs $(26.7 \%)$, least number of patients were observed below the age of 20 yrs (15.8\%) (Fig. 2).

Out of 183 endotracheal aspirates cultured, no growth was observed in 36 samples and was considered bacteriologically sterile. Growth was observed in 147 samples out of which in only 102 samples significant numbers of colonies were observed

Acinetobacter baumanii was the most common isolate observed (31.3\%) followed by Klebsiella pneumonia (24.5\%). In early onset VAP $K$ pneumonia was the predominant isolate whereas in late onset VAP A. baumanii was the predominant. $S$ aureus was most commonly isolated from late onset VAP cases.

Multidrug resistance was commonly observed in $A$. baumanii with majority of the isolates (40.6\%) being resistant to Imipenem. $K$. pnuетоnia and E. coli were absolutely resistant to amoxyclav (100\%) and also showed high resistance towards ceftriaxone (92\% and $80 \%$ ). Tigecycline has shown the highest sensitivity in enterobacteriace members whereas polymyxin group of antibiotics have shown the highest sensitivity in non fermenting gram negative bacilli. Among Staphylococcus aureus isolates, 
MRSA was observed in $66.6 \%$ of the patients and MSSA in the remaining, All the S.aureus were sensitive to vancomycin and linezolid.

VAP is one of the major complications in the ICU patients, the mortality rates from which can range from 25 to $50 \%{ }^{6}$. The bacteriological approach for the management of VAP avoids the problem of over treatment by separating colonizers from infecting pathogens.

Several methods to obtain good respiratory samples have been extensively investigated. each technique having its own diagnostic threshold and methodological limitations. The choice of method depends on expertise, experience, availability and cost. Studies showed that the results of quantitative cultures of endotracheal aspirates were comparable to those using invasive bronchoscopic methods ${ }^{7}$. Our study showed occurrence of VAP to be $55.7 \%$, which was in close agreement with many other studies. In Ranjan et al., ${ }^{8}$ study the incidence of VAP was $57.14 \%$, which is similar to our study.

The patients studied belonged predominantly to the age group of $21-40$ years $(37.1 \%)$. This type of age dominance of VAP was also noticed in Gadani et al., study ${ }^{9}$ where majority of the patients were in the same age group with a mean age of $34 \mathrm{yrs}$. In the present study it was found that $60.1 \%$ of the cases were males and $39.8 \%$ were females. The male predominance can be attributed to high prevalence in males of other co-morbid conditions, risk factors like COPD and high susceptibility to road traffic accidents (RTA) (Table 1-6).

Table.1 Clinical distribution of patients studied

\begin{tabular}{|c|c|c|}
\hline Clinical distribution & Number of Patients & Percentage \\
\hline OP - Poisoning & 49 & $26.7 \%$ \\
\hline Trauma (RTA) & 37 & $20.2 \%$ \\
\hline COPD- Acute exacerbation & 29 & $15.8 \%$ \\
\hline CRF & 21 & $11.4 \%$ \\
\hline Sepsis & 16 & $8.7 \%$ \\
\hline DKA & 13 & $7.1 \%$ \\
\hline Stroke & 8 & $4.3 \%$ \\
\hline Burns & 6 & $3.2 \%$ \\
\hline CAD & 4 & $2.1 \%$ \\
\hline
\end{tabular}

Table.2 Risk factors

\begin{tabular}{|c|c|c|}
\hline Risk Factors & Number of patients & Percentage \\
\hline Diabetes Mellitus & 57 & $31.1 \%$ \\
\hline Advancing age (> 60yrs) & 49 & $26.7 \%$ \\
\hline COPD & 38 & $20.7 \%$ \\
\hline Steroids & 32 & $17.4 \%$ \\
\hline Head Trauma & 31 & $16.9 \%$ \\
\hline CRF & 20 & $10.9 \%$ \\
\hline HIV & 2 & $1 \%$ \\
\hline
\end{tabular}

Table.3 Quantitative culture report 


\begin{tabular}{|c|c|c|}
\hline Quantitative culture report & Frequency in patients & Percentage \\
\hline No organism grown & 36 & $19.6 \%$ \\
\hline Significant growth $\left(>\mathbf{1 0}^{\mathbf{5}} \mathbf{c f u} / \mathbf{m l}\right)$ & 102 & $55.7 \%$ \\
\hline Insignificant growth $\left(<\mathbf{1 0}^{\mathbf{5}} \mathbf{c f u} / \mathbf{m l}\right)$ & 45 & $24.5 \%$ \\
\hline
\end{tabular}

Table.4 Categorization - VAP

\begin{tabular}{|c|c|}
\hline Categorization & Frequency \\
\hline Early Onset & $32.3 \%$ \\
\hline Late Onset & $67.6 \%$ \\
\hline
\end{tabular}

Table.5 Profile of the bacterial isolates

\begin{tabular}{|c|c|c|c|}
\hline Isolates & $\begin{array}{c}\text { Early Onset } \\
(\mathbf{n = 3 3})\end{array}$ & $\begin{array}{c}\text { Late Onset } \\
(\mathbf{n = 6 9 )}\end{array}$ & $\begin{array}{c}\text { Total } \\
(\mathbf{n = 1 0 2})\end{array}$ \\
\hline Acinetobacter baumanni & $8(24.2 \%)$ & $24(34.7 \%)$ & $32(31.3 \%)$ \\
\hline Pseudomonas aeruginosa & $4(12.1 \%)$ & $13(18.8 \%)$ & $17(16.6 \%)$ \\
\hline Klebsiella pneumonia & $11(33.3 \%)$ & $14(20.2 \%)$ & $25(24.5 \%)$ \\
\hline Escherichia coli & $6(18.1 \%)$ & $9(13 \%)$ & $15(14.7 \%)$ \\
\hline Staphylococcus aureus & $2(6 \%)$ & $4(5.7 \%)$ & $6(5.8 \%)$ \\
\hline Enterococcus spp & $2(6 \%)$ & $3(4.3 \%)$ & $5(4.9 \%)$ \\
\hline Stenotrophomonas & - & $2(2.8 \%)$ & $2(1.9 \%)$ \\
\hline
\end{tabular}

Table.6 Antibiotic resistance of GNB

\begin{tabular}{|c|c|c|c|c|c|}
\hline $\begin{array}{c}\text { Antimicrobial } \\
\text { agent }\end{array}$ & $\begin{array}{c}\text { Acinetobacter } \\
\text { baumanni } \\
(\mathbf{n = 3 2})\end{array}$ & $\begin{array}{c}\text { Pseudomonas } \\
\text { aeroginosa } \\
(\mathbf{n = 1 7})\end{array}$ & $\begin{array}{c}\text { Klebsiella } \\
\text { pneumonia } \\
(\mathbf{n = 2 5})\end{array}$ & $\begin{array}{c}\text { Escherichia } \\
\text { coli } \\
(\mathbf{n = 1 5})\end{array}$ & $\begin{array}{c}\text { Stenotrophomonas } \\
(\mathbf{n = 2})\end{array}$ \\
\hline Amikacin & $23(71.8 \%)$ & $6(35.2 \%)$ & $10(40 \%)$ & $5(33.3 \%)$ & $1(50 \%)$ \\
\hline Ceftazidine & $32(100 \%)$ & $14(82.3 \%)$ & - & - & $2(100 \%)$ \\
\hline Imipenem & $13(40.6 \%)$ & $4(23.5 \%)$ & $3(12 \%)$ & $2(13.3 \%)$ & $2(100 \%)$ \\
\hline $\begin{array}{c}\text { Piperacillin } \\
\text { tazobactum }\end{array}$ & $24(75 \%)$ & $11(64.7 \%)$ & $14(56 \%)$ & $8(53.3 \%)$ & $1(50 \%)$ \\
\hline Amoxyclav & $32(100 \%)$ & - & $25(100 \%)$ & $15(100 \%)$ & - \\
\hline Doxycycline & $15(46.8 \%)$ & - & $13(52 \%)$ & $7(46.6 \%)$ & $0(0 \%)$ \\
\hline Ciprofloxacin & $26(81.2 \%)$ & $8(47 \%)$ & $16(64 \%)$ & $8(53.3 \%)$ & $0(0 \%)$ \\
\hline Tigecycline & $4(12.5 \%)$ & - & $0(0 \%)$ & $0(0 \%)$ & $0(0 \%)$ \\
\hline Colistin & $2(6.2 \%)$ & $0(0 \%)$ & - & - & - \\
\hline Cotrimoxazole & $23(71.8 \%)$ & - & $20(80 \%)$ & $13(86.6 \%)$ & $0(0 \%)$ \\
\hline Polymyxin B & - & $0(0 \%)$ & - & - & - \\
\hline Ceftriaxone & - & - & $23(92 \%)$ & $12(80 \%)$ & - \\
\hline
\end{tabular}

Fig.1 Gram Staining from endotracheal aspirate showing Pus cells \& 


\section{Gram negative coccobacilli}

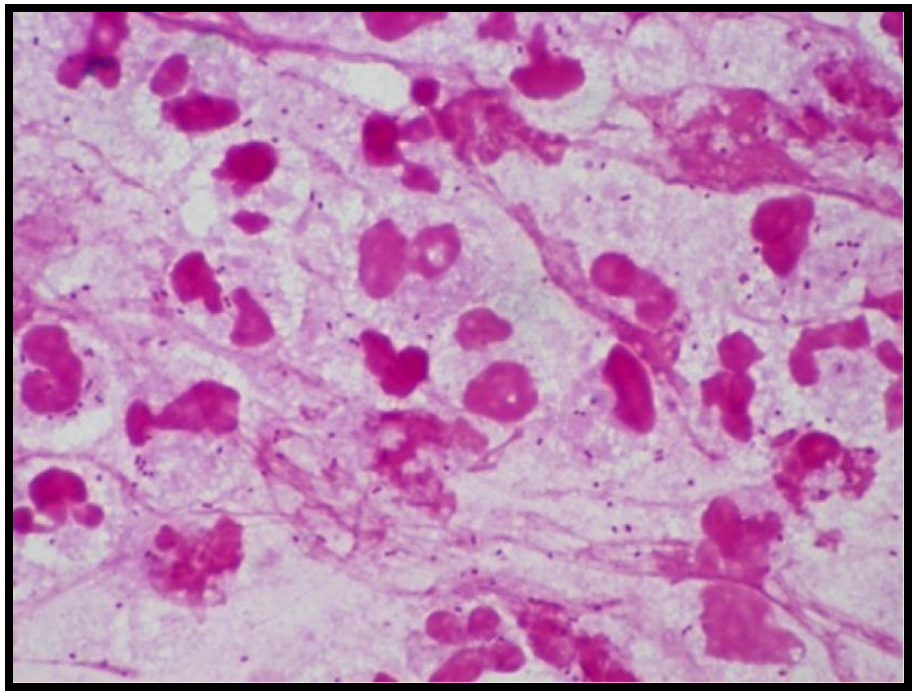

Fig.2 Age distribution

\section{Age distribution}

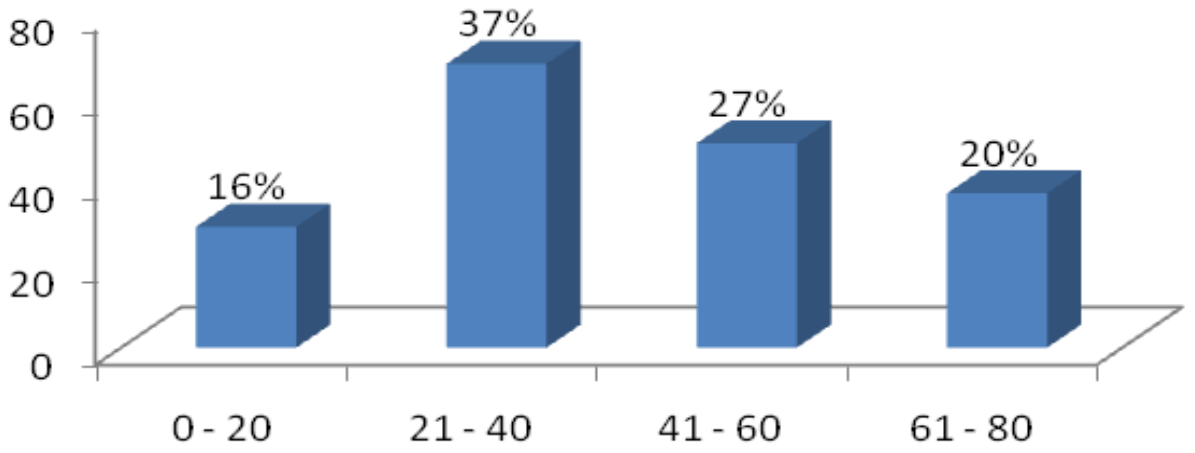

In our study, about $31.1 \%$ of the patients had associated Diabetes mellitus, $26.7 \%$ were in advancing age ( $>60$ years), $20.7 \%$ had COPD and $16.9 \%$ had suffered head trauma. These conditions predispose to colonisation and pneumonia because of disease associated impairment in host defense function. ${ }^{10}$ These were considered as important risk factors for the development of VAP, as shown by various studies. $^{11}$

In our study, the incidence of aerobic gramnegative bacteria was $89.2 \%$ and this high incidence correlated with other studies. ${ }^{7}$ The predominant aerobic gram negative bacteria are Acinetobacter spp. and Klebsiella spp. followed by other aerobic gram negative bacteria like Escherichia coli and 


\section{Pseudomonas aeruginosa.}

In our study we found a significantly high percentage of Acinetobacter baumanni (31.3\%), which was the most predominant organism followed by Klebsiella which is similar to other studies like Kumari et al., ${ }^{12}$ and Singhal et al., ${ }^{4}$

The third common isolate in the present study was Pseudomonas aeruginosa accounting to $16.6 \%$ of the total isolates. Our findings are intermediate between Tripathy et al., ${ }^{13}$ and Kumari et al., ${ }^{12}$ study where the isolation rate was $11 \%$ and $21.5 \%$ respectively. In Tripathy et $a l .,^{13}$ it is the fourth common isolate whereas in Kumari et al., ${ }^{12}$ study it is the second most common isolate

In the present study gram-positive isolates constitute only $10.7 \%$ of the total isolates. The Gram positive isolates were Staphylococcus aureus and Enterococcus spp accounting for $5.8 \%$ and $4.9 \%$ respectively. In a study conducted by Kumari et al., ${ }^{12}$ the isolation rate of $S$ aureus was $7.8 \%$, which was close to our findings. In a study conducted by Singhal et al. ${ }^{4} S$ aureus was the least common organism isolated

In the present study Acinetobacter baumanii was highly resistant to most commonly used empirical drugs like ceftazidine $(100 \%)$, ciprofloxacin (81.2\%), piperacillin/ tazobactum (75\%) and amikacin (71.8\%). These findings are similar in other studies too $^{14}$. In the present study Imipenem resistance was noted to an account of $40.6 \%$ similar to Joseph et al., study ${ }^{14}$.

In the present study Acinetobacter baumanii was least resistant to colistin (6.2\%) followed by tigecycline (12.5\%). Colistin is an age old drug which has been abandoned decades back because of its potential nephrotoxicity but with the recent increase in the carbapenem resistant Acinetobacter isolates, this drug has gained its importance as a potential therapeutic agent despite its toxicity.

Pseudomonas was highly resistant to ceftazidine, the anti-pseudomonal cephalosporin with $82.3 \%$ of the isolates being resistant.

The anti pseudomonal penicillin which is most commonly used in clinical practice is piperacillin/tazobactum, our study shows that $64.7 \%$ of the isolates are resistant to this drug which is in accordance with Modi et al., ${ }^{15}$ study showing resistance of $57 \%$.

All the pseudomonal isolates in our study were uniformly sensitive to Polymyxin B making this antibiotic as a highly effective drug against these infections.

In the present study gram positive isolates constituted only a minority of the total isolates with Staphylococcus aureus showing predominance. Two thirds of the $S$ aureus isolates are cefoxitin resistant (MRSA). All the Staphylococci are uniformly sensitive to vancomycin, linezolid and tigecycline

To conclude, Ventilator associated pneumonia continuous to be an important challenge to the critical care physician as well as microbiologist both for treatment and diagnosis.

A high index of clinical suspicion along with early and prompt diagnosis by using various parameters that include quantitative endotracheal cultures is of utmost importance in appropriate management of the patients, which further decrease the mortality and morbidity along with decrease in the cost associated with the treatment of these infections.

\section{References}


1. Richards, M. J., J. R. Edwards, D. H. Culver, R. P. Gaynes, et al., 1999. Nosocomial infections in medical intensive care units in the United States. Crit. Care Med. 27

2. Baker, A. M., J. W. Meredith, and E. F. Haponik. 1996. Pneumonia in intubated trauma patients. Microbiology and outcomes. Am. J. Respir. Crit. Care Med. 153: 343-349.

3. Rajasekhar T, Anuradha K, Suhasini T, Lakshmi V. The role of quantitative cultures of non-bronchoscopic samples in ventilator associated pneumonia. Indian J Med Microbiol 2006; 24: 10713.

4. Singhal R, Mohanty S, Sood S, Das B, Kapil A. Profile of bacterial isolates from patients with ventilator associated pneumonias in a tertiary care hospital in India. Indian J Med Res 2005; 121: 63 64.

5. Fabregas N, Ewig S, Torres A, ElEbiary M, Ramirez J, Puig de la Bellacasa et al., Clinical diagnosis of ventilator associated pneumonia revisited, comparative validation using immediate post-mortem lung biopsies. Thorax 1999; 54: 867-873.

6. Ewig S, Torres A, El-Ebiary M, Fabregas N, Hernandez C, Gonzalez J et al., Bacterial colonization patterns in mechanically ventilated patients with traumatic and medical head injury. Incidence, risk factors, and association with ventilator-associated pneumonia. AmJ Respir Crit Care Med. 1999; 159: 188-198.

7. Rosenthal VD, Maki DG, Salomao R, Moreno CA, Mehta Y, Higuer F et al., Device- associated nosocomial infections in 55 intensive care units of 8 developing countries. Ann Intern Med 2006; 145: 582-591

8. Neelima Ranjan, K. P. Ranjan, Uma Chaudhary, Dhruva Chaudhry.
Antimicrobial resistance in bacteria causing ventilator-associated pneumonia in a tertiary care hospital: one year prospective study. Int $\mathrm{J}$ Res Med Sci. 2014 Feb; 2(1): 228-233

9. Hina Gadani, Arun V yas, Akhya Kumar. A study of ventilator-associated pneumonia: Incidence, outcome, risk factors and measures to be taken for prevention. Indian J Anaesth. 2010; 54: 535-540.

10. Campbell GD, Niederman MS, Broughton MA, Craven DE, Fein AM, Fink MP et al., American Thoracic Society. Hospital-acquired pneumonia in adults: diagnosis, assessment of severity, initial antimicrobial therapy, and preventive strategies. A consensus statement. Am J Respir Crit Care Med 1995; 153: 1711-1725.

11. Girish L. Dandagi. Nosocomial pneumonia in critically ill patients. Lung India 2010; 27: 149-153.

12. Kumari HB, Nagarathna $S$, Chandramuki A. Antimicrobial resistance pattern among aerobic gramnegative bacilli of lower respiratory tract specimens of intensive care unit patients in a neurocentre. Indian J Chest Dis Allied Sci. 2007; 49: 19-22.

13. S Tripathi, G. K. Malik, A Jain, N Kohli. Study of Ventilator Associated Pneumonia in Neonatal Intensive Care Unit: characteristics, risk factors and outcome. Internet Journal of Medical Update 2010 January; 5(1): 12-19.

14. Noyal Mariya Joseph, Sujatha Sistla, Tarun Kumar Dutta- Ventilatorassociated pneumonia in a tertiary care hospital in India: role of multi-drug resistant pathogens. J Infect Dev Ctries 2010; 4: 218-225.

15. Modi Payal, Javadekar Tanuja, Nanda Sandeep, Pandya Neelam. A study on ventilator associated pneumonia in pediatric age group in a tertiary care 
hospital, Vadodara. National journal of medical research 2012: 2(3); 318-321

\section{How to cite this article:}

Mir Salman Ali, Sreekanth Basireddy, Manisha Singh and Vasanti Kabra. 2019. Etiology, Antibiogram and Quantitative Endotracheal Aspirate Cultures in Ventilator Associated Pneumonia Patients in a Tertiary Care Hospital. Int.J.Curr.Microbiol.App.Sci. 8(07): 245-253. doi: https://doi.org/10.20546/ijcmas.2019.807.031 